Mechanical and electrical contact resistance characteristics of a cellular assembly of carbon nanotubes

This content has been downloaded from IOPscience. Please scroll down to see the full text. 2013 Nanotechnology 24015707

(http://iopscience.iop.org/0957-4484/24/1/015707)

View the table of contents for this issue, or go to the journal homepage for more

Download details:

IP Address: 130.56.107.193

This content was downloaded on 19/06/2014 at 04:04

Please note that terms and conditions apply. 


\title{
Mechanical and electrical contact resistance characteristics of a cellular assembly of carbon nanotubes
}

\author{
M S R N Kiran ${ }^{1}$, U Ramamurty ${ }^{1}$ and Abha Misra ${ }^{2}$ \\ ${ }^{1}$ Department of Materials Engineering, Indian Institute of Science, Bangalore-560012, Karnataka, India \\ 2 Department of Instrumentation and Applied Physics, Indian Institute of Science, Bangalore-560012, \\ Karnataka, India \\ E-mail: abha@isu.iisc.ernet.in
}

Received 14 August 2012, in final form 6 November 2012

Published 7 December 2012

Online at stacks.iop.org/Nano/24/015707

\begin{abstract}
We employ nanoindentation coupled with electrical contact resistance measurements for simultaneous characterization of the electrical and mechanical behaviors of a cellular assembly of carbon nanotubes (CNTs). Experimental results reveal two different responses that correspond to relatively dense and porous regions of the cellular structure. Distinct nonlinear electron transport characteristics are observed, which mainly originate from diffusive conductance in the CNT structure. In the denser region, differential conductance shows asymmetric minima at lower bias, implying that conductivity mainly results from bulk tunneling. However, the porous regions show insignificant differential conduction as opposed to the denser region.
\end{abstract}

S] Online supplementary data available from stacks.iop.org/Nano/24/015707/mmedia

(Some figures may appear in colour only in the online journal)

\section{Introduction}

The entanglement among carbon nanotubes (CNTs) in their bulk microstructure gives rise to a cellular like assembly, which has the potential for impact mitigation similar to cellular foams and strain sensing [1-3]. The mechanical properties of these materials have been investigated using either nanoindentation [4] or compression in bulk [5]. In both the cases, coordinated deformation relying on van der Waals forces between CNTs is reported to occur. Furthermore, these weak interactions also allow electrical conduction paths in the three-dimensional assembly of CNTs [1]. The electrical transport properties of individual tubes as well as their interaction with other CNTs have been studied in detail with emphasis on the evaluation of the modification in the density of states at the Fermi surface due to charge tunneling [6-8]. In general, charge transport among interacting/crossed CNTs is driven by thermal heating [7] or defects induced at the atomic sites [6]. Localized transport characteristics of individual CNTs $[9,6]$ due to incorporation of atomic scale defects are often monitored using advanced nano-manipulation tools equipped with in situ electrical measurement techniques such as scanning tunneling microscopy and atomic force microscopy (AFM). The conductance in two crossing CNTs is shown to be power-law dependent on both the temperature and bias voltage [6]. Similar characteristics are also observed in buckled CNTs where nanoscale tunnel junctions are created using AFM [6]. It is shown that the tunneling conductance reduces to zero as the energy (temperature and bias) is also reduced (a conductance dip centered at zero bias) [10]. Moreover, linear or nonlinear electrical $(I-V)$ characteristics at high bias are attributed to the transport parameters such as electron mean-free path, and the presence of a number of shells [11].

Recently, the manipulation capability of scanning electron microscopes (SEMs) has also provided insights into the transport mechanism of the single CNT. Using this technique, Kaul et al [12] have examined the in situ electro-mechanical response of individual carbon nanofibers under uniaxial compression and demonstrated the occurrence 

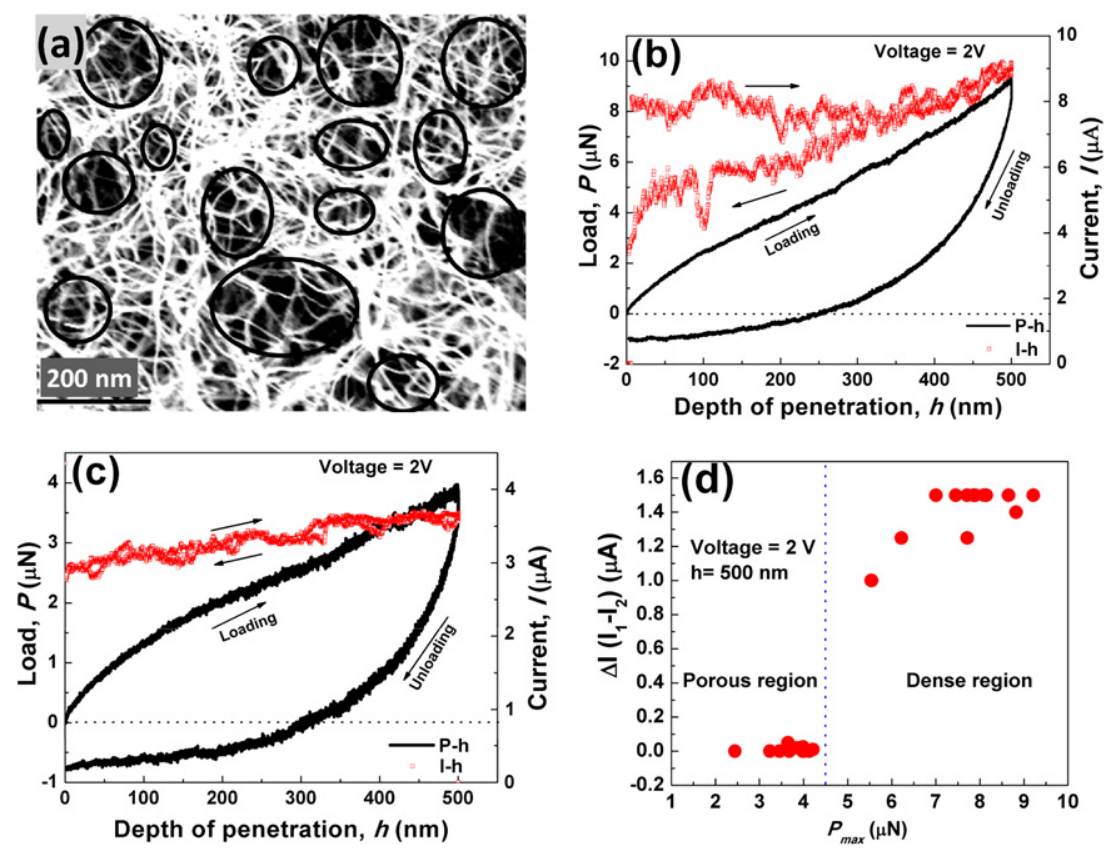

Figure 1. (a) Scanning electron micrograph showing the cellular structure of CNTs. Structural porosity is indicated with circles. Load, $P$, and current, $I$, are plotted against depth of penetration, $h$, obtained from (b) the dense and (c) the porous regions of the CNT structures, respectively. (d) Difference in initial and final currents before and after loading (at zero penetration depth) plotted against the peak load employed for the indentation study.

of severe bending before fracture. Also, Semet et al [13] showed reversible linear and nonlinear characteristics of an individual CNT using an in situ SEM based manipulator. However, at the micron scale, when CNTs in their cellular assembly are compressed, the factors governing the transport mechanism have not yet been examined. The present work addresses this aspect through in situ characterization of the electrical and mechanical responses of cellular CNTs. These studies are performed using a nanoindenter tip capable of simultaneous measurement of the electrical contact resistance (ECR), applied load $(P)$ and depth of penetration $(h)$ while indenting the material. This technique allows the differentiation of dense and porous regions of the cellular structure of CNT, and provides insight into the localized electrical transport in these two regions. Two distinct electrical transport characteristics are noted in these two regions due to the variation in the number of conducting channels. Moreover, charge transport is also shown to vary with the indentation depths and bias voltages, which are again related to the cellular structure of the CNTs.

\section{Experimental details}

Cellular-like CNT mats, $\sim 2 \mathrm{~mm}$ thick, are synthesized using chemical vapor deposition, which was described in an earlier paper [2]. As-grown entangled CNT samples are scraped from the substrate (silicon dioxide) and used as a freestanding integrated CNT assembly. The mechanical properties of these assemblies are measured using a nanoindenter (Triboindenter, Hysitron Inc.) consisting of three-plate capacitive force and displacement transducers that have been modified to provide an electrical path between probe tip and the sample. The CNT sample is fixed to a conductive copper stage using silver conductive adhesive and indented with a boron-doped (B concentration, $N_{d}=1 \times 10^{21} \mathrm{~cm}^{-3}$ ) conducting diamond three-sided pyramidal Berkovich tip (tip radius of $\sim 100 \mathrm{~nm}$ ). The indentation into the CNT sample is performed under depth control mode to maximum depths $\left(h_{\max }\right)$ of 500-2000 nm. In all cases, the loading and unloading rates are maintained at $50 \mathrm{~nm} \mathrm{~s}^{-1}$. During indentation a bias voltage, $V$, is applied to the sample and the current, $I$, is measured through the tip by using a picoammeter/voltage source (Keithley 6487).

The electro-mechanical properties during indentation of the cellular assembly of CNTs are measured in two ways: (1) simultaneous measurement of both $I$ and $P$ at constant $V(=2 \mathrm{~V})$, during loading-unloading of the sample to $h_{\max }=$ $500 \mathrm{~nm}$ and (2) measurement of the $I-V$ characteristic at the peak load $\left(P_{\max }\right)$, while sweeping $V$ between -2 to $+2 \mathrm{~V}$ for $2 \mathrm{~s}$ with $h_{\max }$ varying between 500 and $2000 \mathrm{~nm}$.

\section{Results and discussion}

Figure 1(a) shows the typical microstructure of the cellular assembly of CNTs used in this study. Non-uniform entanglement of the CNT leads to several higher and lower density regions of CNTs (the latter being highlighted with circles). Figures 1(b) and (c) show typical $P-h$ and $I-h$ responses for higher and lower density regions, respectively. (See figure S1 in supplementary information for the reproducibility of these results; available at stacks.iop.org/ Nano/24/015707/mmedia). Two sets of distinct $P-h$ responses are noted. In one set, peak load (at $h_{\max }=500 \mathrm{~nm}$ ) is $\sim 10 \mu \mathrm{N}$ (see figure $1(\mathrm{~b})$ ), whereas it is $\sim 4 \mu \mathrm{N}$ in the other set of 
experiments for the same $h_{\max }$ (see figure 1(c)). This large difference is due to the local variation in the cellular density, which we identify as dense and porous regions. On the basis of statistical analyses, we conclude that the transition between the porous and dense regions is not gradual but occurs with a sharp jump in load. Thus, the peak load values of $\leq 4 \mu \mathrm{N}$ define the porous region, while the dense regions result in significantly higher loads $(\geq 7 \mu \mathrm{N})$ for the same depth of penetration.

The $P-h$ responses have been analyzed to infer the elastic moduli, $E$, of these two regions. In the porous regions, $E=$ $6.8 \pm 2.7 \mathrm{MPa}$, whereas it is $13.2 \pm 1.8 \mathrm{MPa}$ in the dense regions, which is nearly double that of the porous regions. In an earlier work on similar CNT mats, Misra et al [14] showed that $E$ varies approximately linearly with the bulk density of the mats, as given by the following equation:

$$
E=k_{1} d+k_{2} \text {. }
$$

Here, $E$ is the measured elastic modulus of the CNT mat, $d$ is the CNT bulk density and $k_{1,2}$ are the fitting parameters as mentioned in reference [14]. Utilizing this, we estimate that the densities of CNTs in the porous and dense regions are $\sim 0.26$ and $\sim 0.47 \mathrm{~g} \mathrm{~cm}^{-3}$, respectively.

A large hysteresis in the measured $P-h$ responses (figures 1(b) and (c)) indicates that the cellular CNTs are viscoelastic in nature. This leads to mechanical energy absorption. Also, upon unloading a complete recovery was observed, which is similar to earlier reports [1]. An interesting attribute of CNT-nanoindenter tip interaction can be seen upon unloading through an exertion of negative load of $\sim$ $-2 \mu \mathrm{N}$, which is due to van der Waals forces between CNTs and the tip, which lead to a pulling force (tension) on the CNTs by the tip while unloading the sample [15].

Typical $I-h$ curves that are obtained simultaneously with the $P-h$ curves are shown in figures $1(\mathrm{~b})$ and (c). These data are obtained at a constant $V$ of $2 \mathrm{~V}$. Again, we observe two distinct electrical responses. In case of dense regions (figure 1(b)), the loading part of the curves shows a gradual increase in $I$ with $h$. At $h_{\max }, I=9 \mu \mathrm{A}, I$ is found to be as low as $3 \mu \mathrm{A}$ during unloading, when compared with the initial $I$ of $8.5 \mu \mathrm{A}$ during loading. This decrease is probably due to the relatively smaller compressive force by the indenter on CNTs for the same depth (figure 1(b)), which reduces the electrical contact between the CNTs and the indenter, which in turn reduces the number of current conducting channels. However, an insignificant change in the current is observed after loading-unloading in the porous region (figure 1(c)).

These features are further highlighted by examining the difference in $I$ values (before indenting the specimen $\left(I_{1}\right)$ and after a complete unloading $\left.\left(I_{2}\right)\right)$ at $P=0$. Figure 1(d) shows the variation of $\Delta I\left(=I_{1}-I_{2}\right)$ as a function of $P_{\max }$. The data shown in this plot are obtained at various locations in the sample. For $P_{\max } \leq 4 \mu \mathrm{N}$ (porous regions), $\Delta I \sim 0$, and at higher $P_{\max }$ (dense regions) measured current difference is $\Delta I \sim 1.5 \mu \mathrm{A}$. In both the cases, $I$ increases with increasing $P$. This is because of a continuous increase in the contact area between the tip and the CNT mat with increasing $P$, which in turn increases the number of CNTs that make electrical contact with the indenter tip. These observations signify that the transport properties depend on the relative densities of the CNT, but not in a linear fashion. A possible explanation for these distinctly different responses is as follows: the dense region provides a higher number of conducting channels through crossed CNTs, which form tunnel barriers in the microstructure [6]. Therefore, a higher $I$ value is obtained compared to the porous region (lower CNT density). A detailed study of the effect of microstructural changes on the electron transport behavior is under progress.

In order to further elucidate the differences in the charge transport mechanisms, $I-V$ characteristics at various $h_{\max }$ $(0.5-2 \mu \mathrm{m})$ are examined (figure 2). An increase in $I$ with $h_{\text {max }}$ indicates a larger number of tip-CNT and CNT-CNT contacts. In all cases, the $I-V$ curves indicate nonlinear transport, both in the forward and reverse bias conditions $(2 \mathrm{~V})$.

Again, the $I-V$ characteristics obtained from the porous and dense regions are distinctly different. A gradual variation in $I$ from nonlinear (at near zero bias) to the linear with the increase in bias voltage is seen in the dense region (figure 2(a)). This slight nonlinearity is probably due to the presence of fewer weak surface contacts among the CNTs. The inset graph in figure 2(a) shows Ohmic behavior (linear $I-V$ response) of the reference copper plate using the same indentation set-up. In contrast to the dense region, the $I-V$ responses obtained in the porous regions exhibit higher nonlinearity under the same bias conditions (figure 2(b)). It is also interesting to note that the magnitudes of $I$ measured in reverse and forward biases are not the same. A larger $I$ is observed at higher $h_{\max } v i s$-á-vis the lower depths of indentation in both forward and reverse directions. In the dense regions, the magnitude of the forward current is higher than the reverse current (see-figure 2(a)). On the contrary, in the porous regions the magnitude of the reverse current is higher than the forward current (see figure 2(b)). This behavior is probably due to the widening of the barrier [16], regardless of the electron flow direction and the differences in properties of the barrier in different directions. These measurements suggest there are barriers across the crossed tubes in the cellular structure.

The nonlinearity can further reasoned to be due to the complexity in the electron transport associated with the metallic percolation paths in the dense and porous regions [17]. Weak connections created by crossed CNTs are associated with nonlinearity in differential conductance, which is given by $\mathrm{d} I / \mathrm{d} V$ [18]. On the other hand, Ohmic contacts between CNTs are responsible for the linear $I-V$ response. Figure 2(c) illustrates the variation of $\mathrm{d} I / \mathrm{d} V$ with $\mathrm{V}$ for pure $\mathrm{Cu}$ (used for reference) and those for porous and dense regions of the CNT at room temperature. An invariant response exhibited by $\mathrm{Cu}$ indicates Ohmic behavior, while both the dense and porous regions exhibit a nonlinear response. The nonlinear behavior suggests a tunneling, non-Ohmic character for the CNT-CNT contact. At higher bias conditions, conductivity of the CNTs in the dense region is found to be higher than $\mathrm{Cu}$. In addition, conductance in the dense region exhibits asymmetric minima at zero bias, 

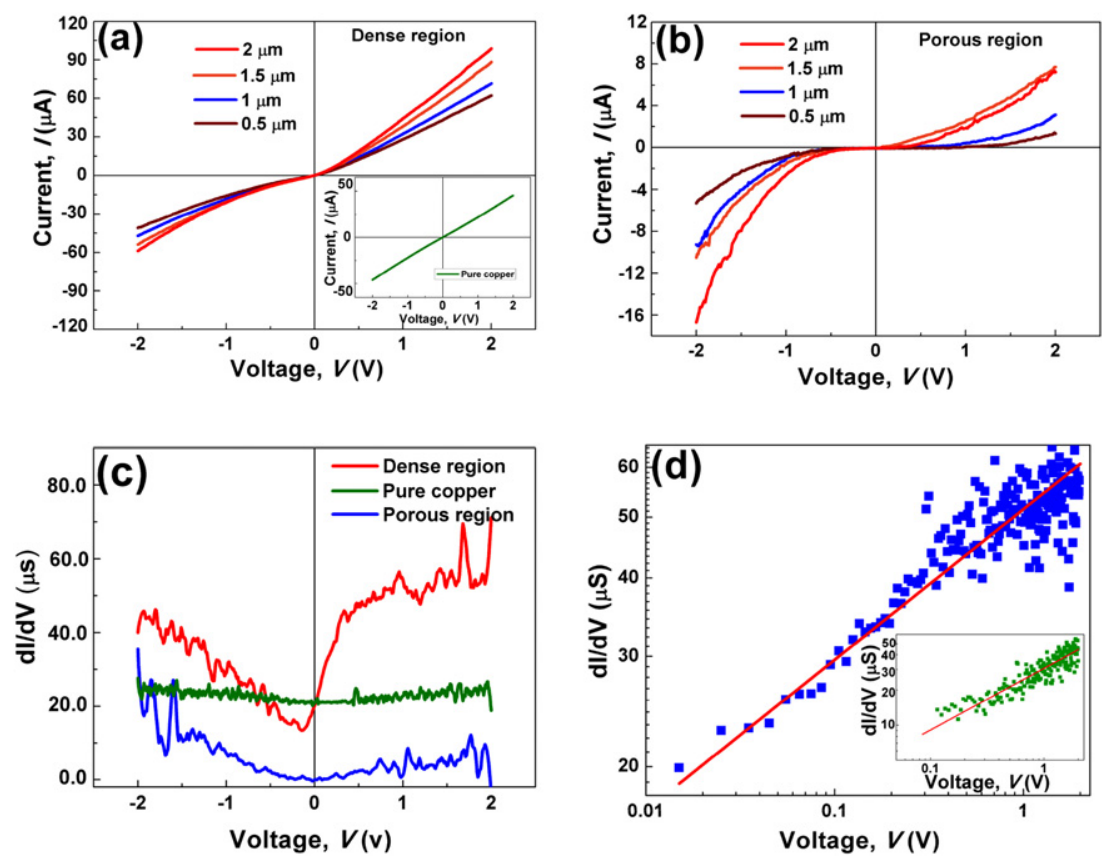

Figure 2. $I-V$ characteristics measured in (a) the dense and (b) the porous regions of the cellular CNT. The inset in (a) shows the Ohmic response measured on a pure copper plate. (c) Differential conductance $(\mathrm{d} I / \mathrm{d} V)$ of the pure copper, dense and porous regions of the CNTs is plotted against bias voltage. (d) Differential conductance for the dense region as a function of $V$ is plotted on a log-log scale (filled squares) for the positive bias region. The solid line is a power-law fit through the data. The inset shows the differential conductance for the dense region as a function of $V$ for the negative bias region.

which can originate from electron-electron interactions and diffusive conduction in the CNTs [19]. However, differential conductance in the porous region represents poor conduction with zero conductivity at zero bias, which is related to the weaker contacts among CNTs as well as the tip of the indenter.

As noted in other reports $[10,16]$, the dense regions show a power-law behavior of differential conductance when plotted against bias voltage on $\log -\log$ scale. The results are in agreement with the earlier prediction for electron tunneling into a Luttinger liquid (LL) from the outer cells of the nanotubes [20]. This type of conductance reflects a power-law behavior of the density of states in the LL, which also supports our observation. In the present case, the exponent is found to be 0.24 (figure 2(d)) for the positive bias, which matches with the theoretically predicted value of 0.24 calculated for the electron tunneling into the bulk-bulk contacts of a LL [21]. However, the exponent value increased to 0.60 , in the negative bias region, which is closer to the theoretically predicted value of 0.65 for the electron tunneling into the end-end of a LL [21]. Thus, it can be surmised that the electron transport in compressed cellular CNT takes place by both end-end and bulk-bulk electron tunneling [21]. Moreover, in a compressed state, electron transport can occur from all cells of the CNT, which enhances the conductance in the dense region of the structure significantly [20]. Therefore, the observed conductance in the dense region is found to be higher than for copper. Furthermore, the mechanical compression induced bending of CNTs lowers the barrier to tunnel the charge carriers. In contrast, the porous regions show an approximate power-law dependence on bias voltage with an exponent of 0.82. Bockrath et al [21] have reported an exponent of 0.87 for CNT ropes, which is similar to that estimated in this study. They attribute this exponent to the presence of extrinsic defects in CNTs. In addition, there is an inverse dependence on the number of conducting channels with the exponent [22], which further explains the higher conductivity in the dense regions as opposed to the porous regions. In our study a direct correlation between the exponent and the applied pressure cannot be interpreted as suggested by Monteverde et al [23]. In the work reported in [23], the number of conducting channels in a rope like single-walled CNT was shown to depend on the applied pressure, which, unlike our work (a few $\mathrm{MPa}$ ) was several $\mathrm{GPa}$, and an inverse relation between pressure and the exponent was inferred. This discrepancy warrants a detailed study of the transport properties for distinguishing the phenomena that control the electron transport in entangled MWCNT foams under various parameters, such as low pressure, biasing conditions, CNT-CNT contact configurations, etc.

\section{Conclusions}

The in situ electrical and mechanical properties of a cellular assembly of CNTs are measured using ECR equipped nanoindentation. Measurements of both properties are shown to exhibit two distinct responses in the cellular structure. Varying peak forces are measured during compression at maximum applied displacement, which signifies the dense and porous regions in the structure. In addition, electrical transport shows nonlinear current-voltage characteristics and flow of irreversible and reversible currents in the dense and 
porous regions, respectively. These differences are attributed to the charge accumulation in the compressed state of the CNT and current flow through barriers between CNT-contacting surfaces. A power-law behavior in differential conductance predicts tunneling into the Luttinger liquid from the outer cells of the nanotubes. This study paves the way to understanding the conduction mechanism in CNTs under an applied load at the nanoscale.

\section{Acknowledgments}

MSRNK thank University Grants Commission, Govt of India for providing a Dr D S Kothari Post-Doctoral fellowship.

\section{References}

[1] Cao A, Dickrell P L, Sawyer W G, Ghasemi-Nejhad M N and Ajayan P M 2005 Science 361307

[2] Misra A, Raney J R, De Nardo L, Craig A E and Daraio C 2011 ACS Nano 57713

[3] Misra A, Greer J R and Daraio C 2009 Adv. Mater. 21334

[4] Hutchens S B, Hall L J and Greer J R 2010 Adv. Func. Mater. 201

[5] Raney J R, Misra A and Daraio C 2011 Carbon 493631

[6] Postma H W Ch, Jonge M, Yao Z and Dekker C 2000 Phys. Rev. B 62 R10653

[7] Park J W, Kim J and Yoo K -H 2003 J. Appl. Phys. 934191

[8] Skákalová V, Kaiser A B, Woo Y-S and Roth S 2006 Phys. Rev. B 74085403
[9] Orlikowski D, Nardelli M B, Bernholc J and Roland C 2000 Phys. Rev. B 6114194

[10] Gao B, Komnik A, Egger R, Glattli D C and Bachtold A 2004 Phys. Rev. Lett. 92216804

[11] Bourlon B, Miko C, Forr'o L, Glattli D C and Bachtold A 2006 Semicond. Sci. Technol. 21 S33

[12] Kaul A B, Megerian K G, Jennings A T and Greer J R 2010 Nanotechnology 21315501

[13] Semet V, Binh V T, Guillot D, Teo K B K, Chowalla M, Amaratunga G A J, Milne W I, Legagneux P and Pribat D 2005 Appl. Phys. Lett. 87223103

[14] Misra A, Raney J R, Craig A E and Daraio C 2011 Nanotechnology 22425705

[15] Mesarovic S Dj, McCarter C M, Bahr D F, Radhakrishnan H, Richards R F, Richards C D, McClain D and Jiao J 2007 Scripta Mater. 56157

[16] Graugnard E, Pablo P J, Walsh B, Ghosh A W, Datta S and Reifenberger R 2001 Phys. Rev. B 64125407

[17] Topinka M A, Rowell M W, Goldhaber-Gordon D, McGehee M D, Hecht D S and Gruner G 2009 Nano. Lett. 91866

[18] Cassell A M, Li J, Stevens R M D, Koehne J E, Delzeit L, $\mathrm{Ng} \mathrm{H}$ T, Ye Q, Han J and Meyyappan M 2004 Appl. Phys. Lett. 852364

[19] Davydov D N, Li J, Shelimov K B, Haslett T L and Moskovits M 2000 J. Appl. Phys. 887205

[20] Mendoza D, Morales F and Escudero R 2004 Solid State Commun. 130317

[21] Bockrath M, Cobden D H, Lu J, Rinzler A G, Smalley R E, Balents L and McEuen P L 1999 Nature 397598

[22] Egger R 1999 Phys. Rev. Lett. 835547

[23] Monteverde M and Nuńẽz-Regueiro M 2005 Phys. Rev. Lett. 94235501 